\title{
Properties of a spinal somatosensory evoked potential recorded in man
}

\author{
E. E L - NEG A M Y A N D E. M. S E D G W I C K \\ From the Wessex Neurological Centre, Southampton General Hospital, Southampton
}

SUMMARY Somatosensory evoked potentials were recorded from the skin overlying the cervical and lumbar spinal cord of man after stimulation of median and tibial nerves respectively. The early negative component (N11) of the cervical potential and the negative lumbar potential (N14) were studied. The spatial properties of N11 and N14 indicate a spinal cord origin. Evidence partly from threshold studies, shows that the low threshold cutaneous afferent fibres were responsible for activating the generators of the potentials. A conditioning test stimulation procedure supports a postsynaptic generator. It is concluded that N11 and N14 have properties similar to the cord dorsum potentials recorded in animals and probably have the same generator, the neurones of the dorsal horn.

A potential recorded from the dorsum of cat spinal cord after dorsal root stimulation was first described by Gasser and Graham (1933). Subsequent investigation showed that it is made up of several components, the first being negative and called N1 (Bernhard, 1952, 1953). This is generated postsynaptically by impulses in low threshold cutaneous afferent fibres whose collaterals extend some distance rostral and caudal to the root entry zone and engage dorsal horn cells (Bernhard, 1952, 1953; Bernhard et al., 1953; Bernhard and Widen, 1953; Willis et al., 1973).

Similar potentials have been recorded from the cord dorsum of monkey (Beall et al., 1977) and man (Ertekin, 1976; Shimoji et al., 1976; Shimoji et al., 1977). The application of epidural or direct cord dorsum electrodes in man, however, carries risks which preclude their use except in special circumstances.

Recently, however, using signal averaging techniques, a complex negative potential has been recorded from electrodes on the skin over the cervical and lumbar enlargements of the cord after stimulation of appropriate nerves (Liberson et al., 1966; Cracco, 1973; Matthews et al., 1974). The technique is easily and safely applied and is becoming a useful investigation in the clinical neurophysiology laboratory (Small, 1975). Clinically correct interpretations, however, depend upon a

Address for reprint requests: Dr E. M. Sedgwick, Wessex Neurological Centre, Southampton General Hospital, Southampton SO1 4XY. Accepted 18 April 1978

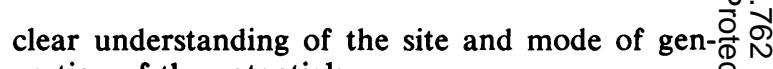

eration of the potentials.

The experiments described here seek to estab- $\stackrel{\mathbb{Q}}{\stackrel{2}{2}}$ lish that an early negative wave recorded from the skin surface is generated in the dorsal horn of the spinal cord by impulses in low threshold cutaneouso क्ष afferents. Some of the properties of this potential suggest that it is equivalent to the $\mathrm{N} 1$ of the cord dorsum potential seen in animals.

\section{Subjects and methods}

Thirty-one normal subjects gave their informed consent and were prepared for recording with silver-silver chloride discs $7 \mathrm{~mm}$ diameter attached over the scalp according to the 10-20 electrode placing system and over the spinal column (Jasper, 1958). Electrodes placed over the spinal column are named according to the vertebral level at which they are attached-for example, the electrode at the seventh cervical level (Cv7) was fixed just rostral to the vertebra prominens, and other dorsal spines were counted rostral or caudal from this point. Fixation was by collodion, and standard techniques ensured that all electrode resistances were less than $5000 \mathrm{ohms}$ and usually less than 2000 ohms (Cooper et al., 1974). Stimulation was through silver-silver chloride discs placed over the peripheral nerves and a large metal plate $60 \times 35 \mathrm{~mm}$ placed on the opposite side of the limb. Devices stimulators were used to deliver pulses of $0.2 \mathrm{~ms}$ at three times sensory threshold 
at a rate of $2 / \mathrm{s}$. The subject was earthed by a conducting band placed around the limb proximal to the stimulating electrodes.

\section{SIGNAL ANALYSIS}

The signal was amplified 40000 times by amplifiers with a bandwidth of $8-10 \mathrm{kHz}$ and fed into the analogue inputs of a PDP 12 computer either on line or from an FM PI 6204 tape-recorder (bandwidth $0-10 \mathrm{kHz}$ ). The gain of the system was checked on each occasion by a calibration signal from a Devices calibrator. The gain factor was later used in the analysis. The stimulator pulse triggered the averaging program and 300 sweeps were averaged at 10 points $/ \mathrm{ms}$. The averaging program (ECOGB) and the analysis program (ECAN) by which the waveforms were smoothed and plotted, were kindly supplied by Dr A. R. D. Thornton, Institute of Sound and Vibration Research, University of Southampton. Amplitude and latency measurements were obtained by using cursors on the computer display and a section of the ECAN program which typed the amplitude and latency of turning points in the waveform. Measurements were also made by eye from computer-produced plots of the averaged potentials.

Recording was in accordance with the clinical neurophysiological practice such that a negativity at the "active" or "black" lead produced an upgoing deflection on the traces. Complete relaxation of the subject was necessary and achieved in all but two who were excluded from the study. The recorded signal was monitored continuously to check for EMG activity or other sources of noise and runs could be terminated if the interference was excessive.

These procedures on normal volunteers and on patients have the approval of the hospital ethical committees.

\section{Results}

CERVICAL SOMATOSENSORY EVOKED RESPONSE

Figure 1 shows the cervical somatosensory evoked potentials recorded from different locations in one subject after stimulation of the median nerve at the wrist with $0.2 \mathrm{~ms}$ pulses of voltage three times sensory threshold. The upper trace, from $\mathbf{C v} 2-F_{\mathbf{z}}$, shows an early waveform composed of three peaks which are consistently seen in all subjects. These peaks or waves are referred to by their polarity and latency and are, therefore, N11, N13, and N14. The subsequent events are of cerebral origin and will not be considered further. The second trace from Cv7-Fz is of the same form but shows an earlier peak at $9 \mathrm{~ms}$ (N9). In the third

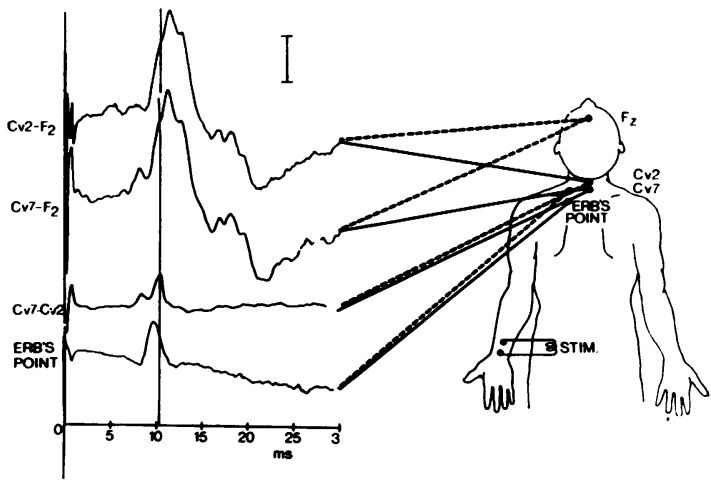

Fig. 1. Cervical somatosensory evoked potentials after median nerve stimulation are shown from different electrode sites using $F_{z}$ as a reference. The vertical line is drawn through the peaks of the N11 potential. $C \nu=$ Cervical and $F_{z}=$ midfrontal electrode placements. Upper trace: from $C \nu 2-F_{z}$ shows the three negative peaks, N11, N13, and N14; second trace: from $C \nu 7-F_{z}$ shows the early N9 potential in addition to the three subsequent peaks; third trace: from $C v 7-C v 2$ shows only the early N9 and N11 potentials, the later components are not recorded; bottom trace: from electrodes at Erb's point which record the ascending volley of impulses in the brachial plexus. This volley passes Erb's point after the N9 peak and before the N11 peak. Amplitude calibration bar: $2 \mu \mathrm{V}$. In this and all subsequent figures a negativity at the active electrode, shown by a solid line, results in an upward deflection.

trace a recording from $\mathrm{Cv} 7-\mathrm{Cv} 2$ shows a simple waveform consisting of the $\mathrm{N} 9$ and $\mathrm{N} 11$ waves followed by a slow positive wave that is just discernible but there are no negative waves after N11. The vertical line was drawn through the peak of N11.

Timing these events with respect to the afferent volley was done by recording the compound action potential as it passed two electrodes, $20 \mathrm{~mm}$ apart, placed on the skin at Erb's point. It can be seen that this peak occurs after N9 but before N11. Jones (1977) has indicated that N9 is a far field potential generated by the compound action potential in the medial cord of the brachial plexus in the lower axilla. Jones considers the advancing potential as a dipole with a positive front which is "seen" by the $F_{z}$ reference electrode. A positivity at this electrode would produce an upward deflection in the trace.

The N11 peak occurs an average of $2.3 \mathrm{~ms}$ after N9 and also after the compound action potential has passed Erb's point. It must, therefore, be generated at a site not very far proximal and the most likely structure is the spinal cord. 
SPATIAL DISTRIBUTION OF N11

It is well known that averaging techniques enable potentials to be recorded at some distance from their generators. Evidence from two types of experiments shows that $\mathrm{N} 11$ is generated at the spinal segmental level.

The site at which the potential is maximal gives an indication of the location of the generator and Fig. 2 shows the results of an experiment in which the cervical somatosensory evoked response was recorded from a number of sites against a reference electrode at $F_{z}$. The vertical line identifies N11 in this subject and the accompanying plots of N11 amplitude against location of the active electrode show clearly that the wave was at its maximum amplitude at or near Cv7. The latency of this peak is constant at all recording sites. The other components, N13 and N14, can be seen to

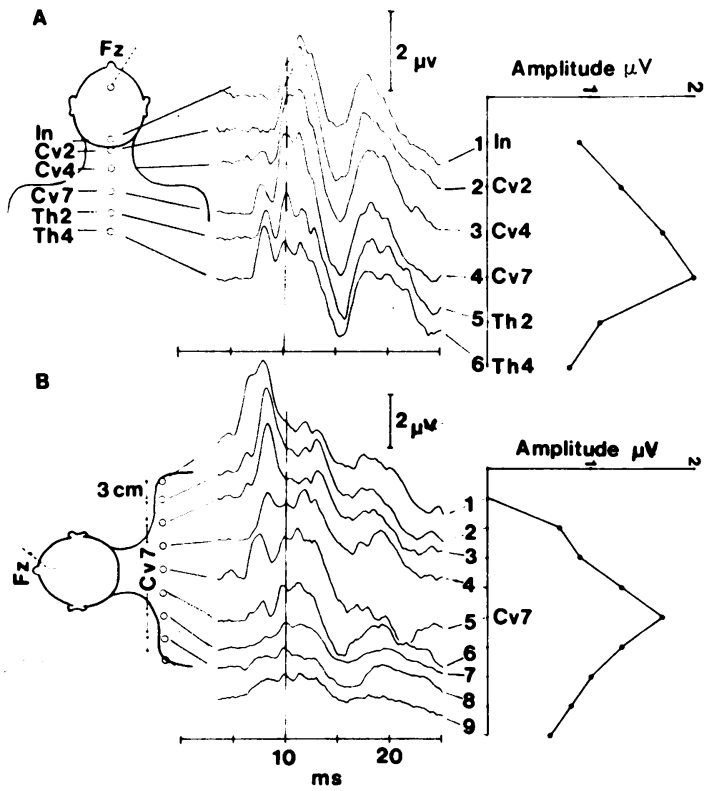

Fig. 2. The somatosensory evoked potential was recorded from several sites using $F_{z}$ as the reference. In $A$ the potentials from the inion (In) cervical $(C v)$, and thoracic levels are shown. N11 is identified by a vertical line through its peak, and the amplitude of the N11 peak is plotted against the recording site alongside the traces. N11 has its greatest amplitude when recorded from $C \nu 7$. In $B$ the recording sites were across the shoulders and N11 is marked by $a$ vertical line. $N 11$ had its maximal amplitude in the midline at $C v 7$. In this series of recordings the high amplitude early waves derived from the right shoulder result from the action potentials in the brachial plexus as the right median nerve was stimulated. Calibration bar $2 \mu \mathrm{V}$. have a different spatial distribution which will be considered in a later publication.

Recording from five patients with clinically complete traumatic spinal cord section at Cv5 or Cv6 showed both N9 and N11 waves. The potential from one subject is shown in Fig. 3. This patient has a complete traumatic cord lesion at the Cv5 level but both the N9 and N11 components can be identified from this $\mathrm{Cv} 7-\mathrm{F}_{\mathrm{z}}$ recording. The upper trace in Fig. 3 shows an attempt to record a cortical potential but its absence confirms the completeness of the cervical cord lesion.

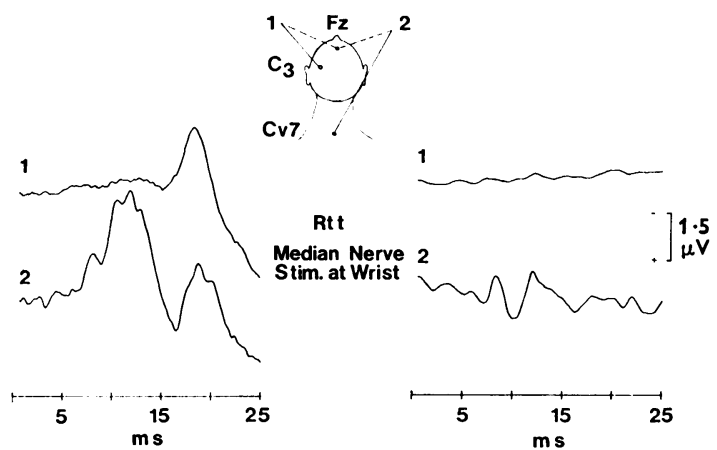

Fig. 3. The two traces on the left show scalp (upper) and cervical somatosensory evoked potentials after median nerve stimulation in a normal subject. On the right the upper trace shows no scalp potential in a subject with a clinically complete traumatic spinal cord lesion at C V5. The lower trace shows a peak corresponding to N9, the brachial plexus potential, and a second negative peak corresponding to N11. Calibration bar $1.5 \mu \mathrm{V}$.

\section{MICROREFLEXES}

It could be argued that the potentials represent microreflexes (Bickford, 1964, 1966). Cracco and Bickford (1968) studied the microreflexes evoked by median nerve stimulation in man and found the earliest reflex event occurred $14 \mathrm{~ms}$ after the stimulus. The latency of $\mathrm{N} 11$ is, therefore, too early for it to be a microreflex but, in order to exclude this as a mechanism generating the later waves, recordings were made in subjects who were asked to contract their neck muscles slightly. The procedure should enhance microreflexes but in our subjects no evoked potential was discernible. In one other experiment recordings were taken before and during light anaesthesia with complete muscle relaxation produced by gallamine in a subject before surgery. The evoked potential was present and unchanged after paralysis, thus excluding microreflexes. 
LUMBAR SOMATOSENSORY EVOKED RESPONSE

In experiments in which the recording electrodes were placed over the lumbar and lower thoracic vertebrae with a reference electrode at the inferior angle of the scapula, potentials were detected after stimulation of the tibial nerve in the popliteal fossa. Figure 4 shows the potentials obtained from electrodes placed over lower thoracic and lumbar vertebrae. There are two negative waves-the first, N10, was recorded by the caudal electrodes and a later $\mathrm{N} 14$ recorded at more rostral sites. The distributions of the two waves overlap and electrodes between L2 and Th 12 usually record both waves. The distribution and latencies suggest that $\mathrm{N} 10$ is generated in the roots of the cauda equina and N14 by the cord itself. The spinal cord terminates at the lower level of L1 vertebra and the cord segments receiving tibial nerve afferents (L5, S1, and S2) are situated close to the Th 12 vertebra.

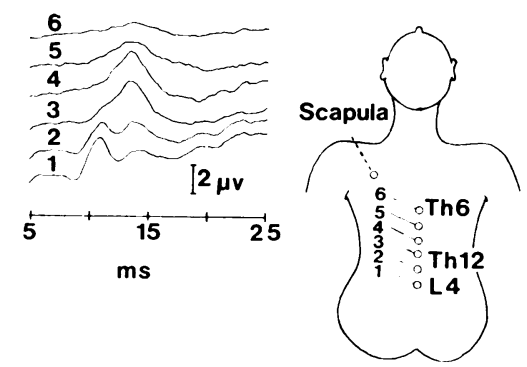

Fig. 4. The potentials evoked after right tibial nerve stimulation at the popliteal fossa are shown from different spinal levels. Traces 1 and 2 from the fourth and second lumbar vertebral levels show an early negative peak, N10; there is also a later peak, N14, seen at its maximum amplitude at Th 12 vertebral level which is the anatomical site of the root entry zone of the tibial nerve fibres. Calibration bar $2 \mu \mathrm{V}$.
FIBRES RESPONSIBLE FOR N11 AND N14

Stimulation of the median nerve at the wrist excites cutaneous and muscle afferents and motor axons. All of these could contribute to N11 and N14 but the evidence indicates that low threshold cutaneous afferents were the only group contributing significantly to the potentials. Cutaneous afferents alone can be excited by stimulating the digits and Fig. 5 shows the results of an experiment in which this was done. The potentials N9, N11, N13, and N14 can be recognised in the recording from $\mathrm{Cv} 7-\mathrm{F}_{\mathrm{z}}$. They are of lower amplitude than those after stimulation at the wrist, as fewer fibres would be stimulated. Their latencies are delayed by 2-3 ms which is accounted for by the conduction time from the digit stimulating electrodes to the wrist.

In a second experiment the median nerve was stimulated at the wrist and the amplitude of the motor (M) response of abductor pollicis brevis gave an indication of the number of motor axons excited by the stimulus. With increasing stimulus strength both the $M$ response and N11 increased in amplitude but at higher strengths N11 began to plateau while the $M$ response curve became steeper (Fig. 6).

The difference of the two recruitment curves indicates that antidromic impulses in the motor fibres, which presumably invade the motoneurones in the cord, did not make a major contribution to the N11 potential.

A similar experiment was carried out with tibial nerve stimulation while recording from soleus muscle. Here both the $M$ response and the Hoffmann $(\mathrm{H})$ reflex due to a volley in the Ia muscle afferents could be recorded (Magladery et al., 1951). As in the case of the median nerve there is a discrepancy between the $M$ response and the amplitude of the N14 lumbar spinal somatosensory

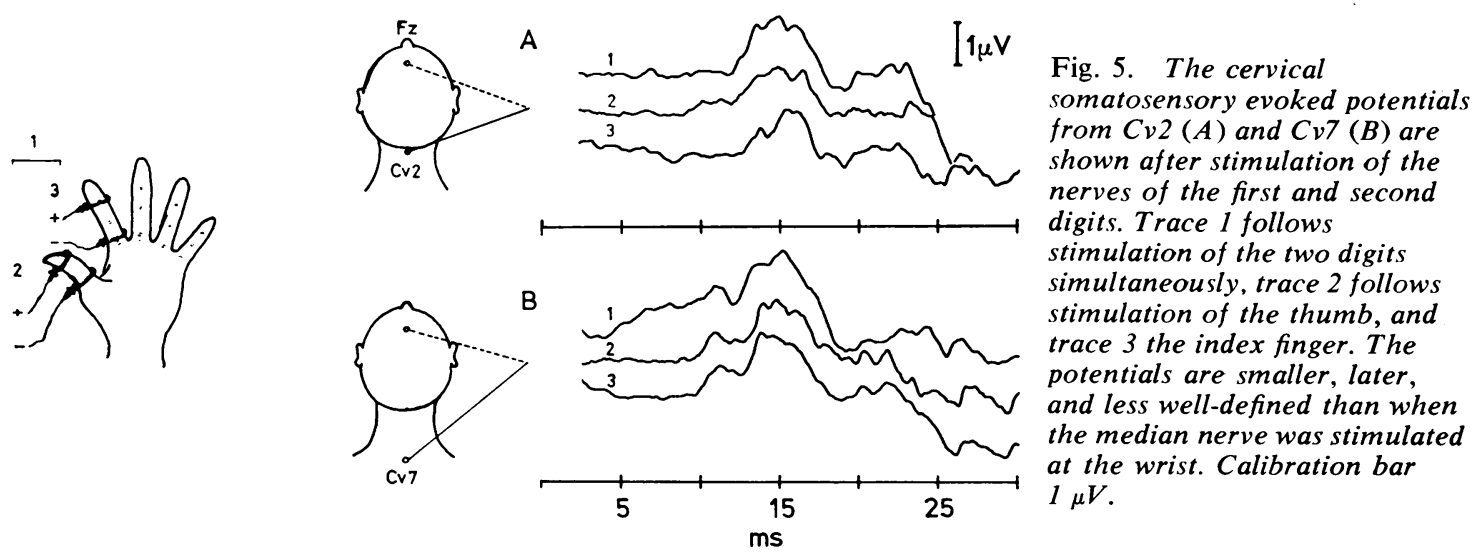




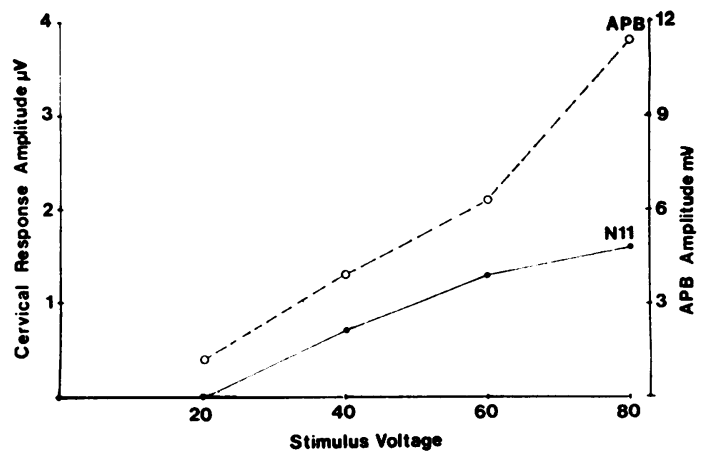

Fig. 6. The amplitude of N11 is shown plotted against increasing stimulus strength to the median nerve at the wrist (- ordinate on left). The stimuli evoked a twitch in the thenar muscles. The amplitude of the electrical response record from skin electrodes over the belly and tendon of abductor pollicis brevis $(A P B)$ is also plotted (-....., ordinate on right). At higher stimulus strengths $N 11$ increases very little while a marked increase in abductor pollicis brevis potential indicates a large increase in the number of motor fibres stimulated.

evoked response (Fig. 7). The N14 recruitment curve can be seen to be at its steepest at a time when few motor axons are activated, and the N14 recruitment is reduced at higher stimulus strengths when the $M$ response is recruiting rapidly.

Further, at a stimulus strength sufficient to excite enough low threshold muscle afferent fibres to produce a maximum $\mathrm{H}$ reflex, the lumbar somatosensory evoked potential was very small indicating that neither the arrival of the muscle afferent volley nor discharge of a significant proportion of the motoneurone pool contribute to the potential.

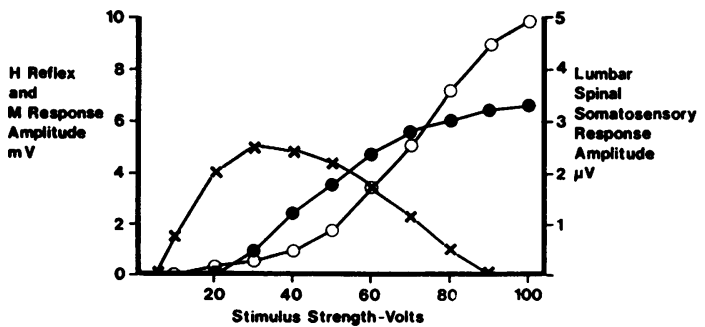

Fig. 7. Stimulation of the tibial nerve in the popliteal fossa with increasing stimulus strengths produces a Hoffmann $(H)$ reflex in soleus muscle $(\mathrm{x}-\mathrm{x})$ which rises to a maximum and falls as the direct motor $(M)$ response $(\mathrm{O}-\mathrm{O})$ increases. The lumbar somatosensory potential (N14 •- $\bullet$ ) is just present when the $H$ reflex is at its maximum and its amplitude begins to saturate at stimulus strengths which are increasing the $M$ response.
N11 AND N14 ARE POSTSYNAPTIC POTENTIALS

Impulses in the low threshold cutaneous afferents will ascend in the dorsal columns and engage synapses of the dorsal horn neurones. Either of these structures could generate the potentials but the following evidence excludes the dorsal columns.

It is accepted that the dorsal columns will respond to high rates of stimulation as do the peripheral nerve fibres. With repetitive stimulation at rates of $10 / \mathrm{s}$ and above, the cervical response alters its form. Figure 8 shows the result of an experiment in which equal paired stimuli were given to the median nerve with different interstimulus intervals. The amplitudes of N9 and N11 are plotted as a percentage of the response size when stimulated once a second. The N9 potential remained stable even at the shortest interval of $10 \mathrm{~ms}$ while the N11 wave was attenuated at intervals less than $60 \mathrm{~ms}$ falling to $20 \%$ of normal at a $10 \mathrm{~ms}$ interval (Fig. 8, left). A similar but slightly steeper recovery curve was obtained from the tibial nerve for the N14 response (Fig. 8, right), but the N10 wave remained steady.

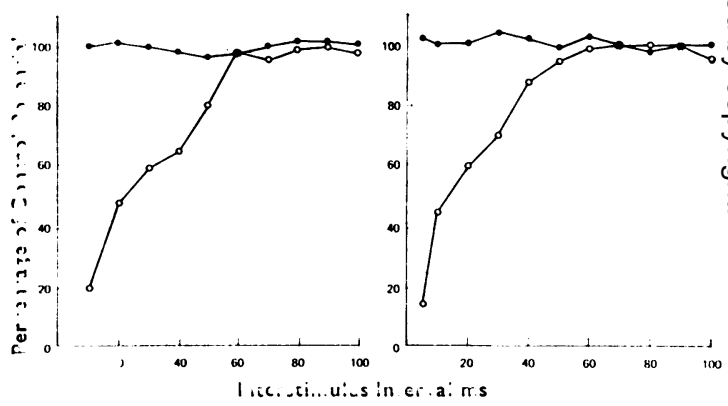

Fig. 8. Two equal stimuli were delivered with a short time interval between. The amplitude of the second spinal somatosensory potential is plotted as a percentage of the first. Left: cervical potentials N9 $-\longrightarrow$ and N11 O- $\bigcirc$ recorded from $C v 7-F_{z}$. Right: lumbar potentials N10 —— and N14 $\bigcirc-\bigcirc$ recorded from $T h 12$ scapular.

\section{Discussion}

A potential originating in the spinal cord of man has been recorded by Magladery et al. (1951), Ertekin (1976), and Shimoji et al. (1976, 1977). This potential is the cord dorsum potential of man and is similar to that recorded in cat by Gasser and Graham (1933) and Bernhard (1952), and in monkey by Beall et al. (1977). The cervical somatosensory potential recorded by surface electrodes is a more complex 
potential with several components but it can be recorded consistently, and our findings concerning the form of the response agree with those of Matthews et al. (1974). With the exception of the N9 component, the origins of the other parts of the response have not been demonstrated conclusively. Cracco (1973) postulated a travelling wave produced by the compound action potential in the dorsal column fibres whereas Matthews et al. (1974) favoured fixed site generators. In these experiments the latency of N11 was constant for any subject regardless of the site of the recording electrode indicating a fixed site generator. Cracco and Cracco (1976) suggested that the potential is a positive "far field" potential recorded from the reference electrode on the scalp, and that this potential is produced at a different generator from the spinal potential but with similar latency.

If the N11 is a positive wave recorded from the $F_{z}$ electrode, it should be present whatever the site of the other electrode so long as this is not on the scalp. If, however, the $\mathrm{N} 11$ is a spinal potential and not recorded by the $F_{z}$ reference electrode, it should get larger as the active electrode is moved nearer the generator. The experiments illustrated in Fig. 2 show that $\mathrm{N} 11$ increased as the active electrode approached the area over the spinal cord root entry zone of the median nerve. The N11 potential could also be recorded from $\mathrm{Cv} 7-$ Cv2 when neither electrode was on the scalp (Fig. 1, trace 3). In this instance the later potentials were absent indicating that either the two electrodes are isoelectric for the late components or that they are positive potentials at $\mathbf{F}_{\mathbf{z}}$.

The spinal somatosensory potential was produced by sensory (digital) nerve stimulation and, when stimulating mixed nerves, the effective fibres had a stimulus threshold greater than that for Ia fibres but equal to or less than the motor axons. The low threshold cutaneous afferent fibres have appropriate thresholds, and it is concluded that synchronous volleys in them ultimately produce the cord potential in man. Jones (1977) has measured their conduction velocity and gives a value of $65-76 \mathrm{~m} / \mathrm{s}$ which is appropriate for low threshold cutaneous afferent fibres.

Consideration of the anatomy of the spinal cord shows many dorsal horn neurones with dendrites running dorsally from the perikaryon and perpendicular to the surface of the cord (Scheibel and Scheibel, 1968). The low threshold cutaneous afferent fibres make synaptic contact with these dendrites and synchronous excitation of such a structural ensemble would be expected to produce a negative potential recorded at the surface. How- ever, a synchronous volley in the dorsal roots, the dorsal column fibres, or the flame-shaped terminal arborisations could also be expected to contribute. Austin and McCouch (1955) were able to identify an early presynaptic component from cord dorsum recordings in cats but it was of very low amplitude and has not been noted by other authors.

The nerve roots and the dorsal column fibres were suggested by Jones (1977) as the generators of N11 in man. If this was the case N11 should follow high rates of stimuli faithfully but the double stimuli experiments show that both the cervical N11 and lumbar N14 potentials are attenuated for up to $60 \mathrm{~ms}$ after a preceding stimulus. The N9 and N10 potentials, however, are reproduced faithfully at interstimulus times as low as $10 \mathrm{~ms}$. The inability to follow high rates of stimuli strongly suggests that $\mathrm{N} 11$ and N14 are generated by postsynaptic structures while the spatial properties of both indicate a spinal cord origin. The first synapse of low threshold cutaneous afferents is on to dorsal horn neurones, and activation of these in animals produces the cord dorsum potential.

It is difficult to escape the conclusion that the surface-recorded N11 and N14 are generated in the dorsal horn of man yet the definitive experiment of comparing surface recordings with those taken from the human cord dorsum at surgery has not been possible. Such a comparison has been made by Happel et al. (1975) who found a very close correspondence in the form of potentials from the two sites although those from the cord dorsum were 10 times greater in amplitude.

A thorough understanding of the generator mechanisms is of practical importance as different but characteristic changes have been reported in multiple sclerosis (Small, 1975) and cervical spondylosis (El-Negamy and Sedgwick, 1978) diseases which are at times difficult to differentiate.

We acknowledge gratefully the assistance given by Dr A. R. D. Thornton with computer programming and Mrs $\mathbf{S}$. Emson who typed the manuscript. The Head of tive Department of Physiology and Biochemistm, University of Southampton, kindly gave $c^{r}$. ported by the Government of the Arab Republic of Egypt.

\section{References}

Austin, G. M., and McCouch, G. P. (1955). Presynaptic component of intermediary cord potential. Journal of Neurophysiology, 18, 441-451.

Beall, J. E., Applebaum, A. E., Foreman, R. D., and Willis, W. D. (1977). Spinal cord potentials evoked by cutaneous afferents in the monkey. Journal of Neurophysiology, 40, 199-211. 
Bernhard, C. G. (1952). The cord dorsum potentials in relation to peripheral source of afferent stimulation. Cold Spring Harbor Symposium in Quantitative Biology, 17, 221-232.

Bernhard, C. G. (1953). The spinal cord potentials in leads from the cord dorsum in relation to peripheral source of afferent stimulation. Acta Physiologica Scandinavica, 29, Supplement 106, 129.

Bernhard, C. G., and Widen, L. (1953). On the origin of the negative and positive cord potentials evoked by low threshold cutaneous fibres. Acta Physiologica Scandinavica, 29, Supplement 106, 42-54.

Bernhard, C. G., Lindblom, U. F., and Ottosson, J. O. (1953). The longitudinal distribution of the negative cord dorsum potential following stimulation of low threshold cutaneous fibres. Acta Physiologica Scandinavica, 29, Supplement 106, 170-179.

Bickford, R. G. (1964). Fast motor systems in man: physiopathology of the sonomotor responses. Transactions of the American Neurological Association, 89, 56-58.

Bickford, R. G. (1966). Human microreflexes revealed by computer analysis. Neurology (Minneapolis), 16, 302.

Cooper, R., Osselton, J. W., and Shaw, J. C. (1974). EEG Technology. Second edition. Butterworths: London.

Cracco, R. Q. (1973). Spinal evoked response: peripheral nerve stimulation in man. Electroencephalography and Clinical Neurophysiology, 35, 379386.

Cracco, R. Q., and Bickford, R. G. (1968). Somatomotor and somatosensory evoked responses. Archives of Neurology (Chicago), 18, 52-68.

Cracco, R. Q., and Cracco, J. B. (1976). Somatosensory evoked potentials in man: far field potentials. Electroencephalography and Clinical Neurophysiology, 41, 460-466.

El-Negamy, E., and Sedgwick, E. M. (1978). The cervical somato-sensory potentials in patients with cervical spondylosis. Electroencephalography and Clinical Neurophysiology. In press.

Ertekin, C. (1976). Studies on the human evoked electrospinogram. Acta Neurologica Scandinavica, 53, 3-20.

Gasser, H. S., and Graham, H. T. (1933). Potentials produced in the spinal cord by stimulation of dorsal roots. American Journal of Physiology, 103, 303320.

Happel, L. T., Le Blanc, H. J., and Kline, D. G. (1975). Spinal cord potentials evoked by peripheral nerve stimulation. Electroencephalography and Clinical Neurophysiology, 38, 349-354.

Jasper, H. H. (1958). Report of the Committee on methods of clinical examination in electroencephalography. Electroencephalography and Clinical Neurophysiology, 10, 370-375.

Jones, S. J. (1977). Short latency potentials recorded from the neck and scalp following median nerve stimulation in man. Electroencephalography and Clinical Neurophysiology, 43, 853-863.

Liberson, W. T., Gratzer, M., Zales, M., and Wrabinski, B. (1966). Comparison of conduction velocities of motor and sensory fibres determined by different methods. Archives of Physical Medicine and Rehabilitation, 47, 17-23.

Magladery, J. W., Porter, W. E., Pack, A. M., and Teasdale, R. D. (1951). Electrophysiological studies of nerve and reflex activity in normal man. IV The two neurone reflex and identification of certain action potentials from spinal roots and cord. Bulletin of the Johns Hopkins Hospital, 88, 499519.

Matthews, W. B., Beauchamp, M., and Small, D. G. (1974). Cervical somato-sensory evoked responses in man. Nature, 252, 230-232.

Scheibel, M. E., and Scheibel, A. B. (1968). Terminal axonal pattern in cat spinal cord. II The dorsal horn. Brain Research, 9, 32-58.

Shimoji, K., Matsuki, M., and Shimizu, H. (1977). Wave form characteristics and spatial distribution of evoked spinal electrogram in man. Journal of Neurosurgery, 46, 304-313.

Shimoji, K., Michiko, M., Ito, Y., Masuko, K., Maruyama, M., Iwane, T., and Aida, S. (1976). Interactions of human cord dorsum potential. Journal of Applied Physiology, 40, 79-84.

Small, D. G. (1975). Peripherally evoked spinal cord potential in neurological diagnosis. In Scientific Aids in Hospital Diagnosis, pp. 155-163. Edited by J. P. Nicholson. Plenum Press: New York.

Willis, W. D., Weir, M. A., Skinner, R. D., and Bryan, R. N. (1973). Differential distribution of spinal cord field potentials. Experimental Brain Research, 17, 169-176. 